\title{
Multiple solutions for Robin $(p, q)$-equations plus an indefinite potential and a reaction concave near the origin
}

\author{
Nikolaos S. Papageorgiou ${ }^{1} \cdot$ Andrea Scapellato $^{2}$ \\ Received: 9 October 2019 / Revised: 11 December 2020 / Accepted: 19 January 2021 / \\ Published online: 9 March 2021 \\ (c) The Author(s) 2021, corrected publication 2021
}

\begin{abstract}
We consider a Robin problem driven by the $(p, q)$-Laplacian plus an indefinite potential term. The reaction is either resonant with respect to the principal eigenvalue or $(p-1)$-superlinear but without satisfying the Ambrosetti-Rabinowitz condition. For both cases we show that the problem has at least five nontrivial smooth solutions ordered and with sign information. When $q=2$ (a $(p, 2)$-equation), we show that we can slightly improve the conclusions of the two multiplicity theorems.
\end{abstract}

Keywords $(p, q)$-Laplacian · Indefinite potential · Resonant and superlinear reaction $\cdot$ Nonlinear regularity $\cdot$ Nonlinear maximum principle $\cdot$ Nodal solutions Locally concave term

Mathematics Subject Classification 35J20 - 35J60

$凶 \quad$ Andrea Scapellato

andrea.scapellato@unict.it

Nikolaos S. Papageorgiou

npapg@math.ntua.gr

1 Department of Mathematics, Zografou Campus, National Technical University, Athens 15780, Greece

2 Dipartimento di Matematica e Informatica, Università degli Studi di Catania, Viale Andrea Doria 6, 95125 Catania, Italy 


\section{Introduction}

Let $\Omega \subseteq \mathbb{R}^{N}$ be a bounded domain with a $C^{2}$-boundary $\partial \Omega$. In this paper, we study the following nonlinear, nonhomogeneous Robin problem

$$
\begin{cases}-\Delta_{p} u(z)-\Delta_{q} u(z)+\xi(z)|u(z)|^{p-2} u(z)=f(z, u(z)) & \text { in } \Omega \\ \frac{\partial u}{\partial n_{p q}}+\beta(z)|u|^{p-2} u=0 & \text { on } \partial \Omega\end{cases}
$$

with $1<q<p$. For every $r \in(1, \infty)$, by $\Delta_{r}$ we denote the $r$-Laplace differential operator defined by

$$
\Delta_{r} u=\operatorname{div}\left(|D u|^{r-2} D u\right) \quad \text { for all } u \in W^{1, r}(\Omega) .
$$

In problem (1.1) we have the sum of two such operators. So, the differential operator in (1.1) is not homogeneous and so many of the techniques used in $p$-Laplacian equations, can not be employed here. Equations driven by the sum of two operators of different nature, arise often in the mathematical models of various physical processes. We mention the works of Bahrouni-Rădulescu-Repovš [3] (transonic flow problems), Benci-D’ Avenia-Fortunato-Pisani [5] (quantum physics), Cherfils-Il'yasov [7] (reaction-diffusion systems), Zhikov [34] (nonlinear elasticity theory).

In problem (1.1), in addition to the $(p, q)$-differential operator there is also a potential term $\xi(z)|u|^{p-2} u$, with the potential function $\xi \in L^{\infty}(\Omega)$ being in general indefinite (that is, sign-changing). This means that the left-hand side of (1.1) is not coercive, an additional difficulty in dealing with problem (1.1).

In the reaction (right-hand side of (1.1)), the function $f(z, x)$ is a Carathéodory function (that is, $z \mapsto f(z, x)$ is measurable for all $x \in \mathbb{R}$ and $x \mapsto f(z, x)$ is continuous for a.a. $z \in \Omega$ ). We consider two different cases concerning the growth of $f(z, \cdot)$ as $x \rightarrow \pm \infty$. First we assume that $f(z, \cdot)$ exhibits $(p-1)$-linear growth as $x \rightarrow \pm \infty$ (that is, $f(z, \cdot)$ is asymptotically $(p-1)$-homogeneous). In this case we permit resonance with respect to the principal eigenvalue of $u \mapsto-\Delta_{p} u+\xi(z)|u|^{p-2} u$ with Robin boundary condition. The resonance occurs from the right of the principal eigenvalue $\widehat{\lambda}_{1}(p)$, in the sense that

$$
p F(z, x)-\widehat{\lambda}_{1}(p)|x|^{p} \rightarrow+\infty
$$

uniformly for a.a. $z \in \Omega$, as $x \rightarrow \pm \infty$, with $F(z, x)=\int_{0}^{x} f(z, s) \mathrm{d} s$.

This makes the energy (Euler) functional of the problem unbounded from below (hence noncoercive) and so we can not use the direct method of the calculus of variations. In the second case we assume that $f(z, \cdot)$ is $(p-1)$-superlinear as $x \rightarrow \pm \infty$ 
but without satisfying the usual in such cases Ambrosetti-Rabinowitz condition (the "AR-condition" for short). In both cases we assume that $f(z, \cdot)$ is concave near the origin and also has an oscillatory behavior.

In the boundary condition $\frac{\partial u}{\partial n_{p q}}$ denotes the conormal derivative corresponding to the $(p, q)$-Laplacian. This directional derivative is interpreted using the nonlinear Green's identity (see Papageorgiou-Rădulescu-Repovš [21], Corollary 1.5.16, p. 34) and if $u \in C^{1}(\bar{\Omega})$, then

$$
\frac{\partial u}{\partial n_{p q}}=\left[|D u|^{p-2}+|D u|^{q-2}\right] \frac{\partial u}{\partial n},
$$

with $n(\cdot)$ being the outward unit normal on $\partial \Omega$.

Using variational tools based on the critical point theory together with truncation and comparison techniques, we show that in both cases problem (1.1) has at least five nontrivial smooth solutions which are ordered and we provide sign information for all of them.

The starting point of our work here is the recent paper of Papageorgiou-Scapellato [24] where the authors deal with a generalized version of the classical concave-convex problem, for equations driven only by the $p$-Laplacian. The reaction there is parametric and nonnegative and they prove a bifurcation-type theorem describing the changes in the set of positive solutions as the parameter $\lambda>0$ varies. Here there is no parameter and for this reason we require that $f(z, \cdot)$ changes sign. Moreover, in the present work in addition to constant sign solutions, we also produce nodal (that is, sign-changing) solutions.

Multiplicity results for $(p, q)$-equations with resonant or superlinear reaction, can be found in Candito-Gasiński-Livrea [6], Filippakis-Papageorgiou [8], GasińskiPapageorgiou [10], Gasiński-Winkert [12], Li-Rong-Liang [16], PapageorgiouRădulescu [17,18], Papageorgiou-Scapellato [25], Papageorgiou-Vetro-Vetro [26], Pei-Zhang [29], Sun [31]. Also our work here extends that of Gasiński-Papageorgiou [11], where the authors permit only nonuniform nonresonance for equations driven by the Dirichlet $p$-Laplacian with no potential term. We also mention the very recent work of Vetro [32], where the author examines perturbations (both sublinear and superlinear) of the eigenvalue problem for the operator $u \mapsto-\Delta_{p} u+\xi(z)|u|^{p-2} u$ with Robin boundary condition. Finally we should also mention the works of Amster [2], Papageorgiou-Rădulescu-Repovš [22,23] and Papageorgiou-Zhang [28], where the authors deal with problems involving concave terms. 


\section{Mathematical background-hypotheses}

In the analysis of problem (1.1), the main spaces are the Sobolev space $W^{1, p}(\Omega)$ and the Banach space $C^{1}(\bar{\Omega})$. By $\|\cdot\|$ we denote the norm of $W^{1, p}(\Omega)$ defined by

$$
\|u\|=\left[\|u\|_{p}^{p}+\|D u\|_{p}^{p}\right]^{\frac{1}{p}} \quad \text { for all } u \in W^{1, p}(\Omega)
$$

The space $C^{1}(\bar{\Omega})$ is an ordered Banach space with positive (order) cone $C_{+}=\{u \in$ $C^{1}(\bar{\Omega}): u(z) \geq 0$ for all $\left.z \in \bar{\Omega}\right\}$. This cone has a nonempty interior given by

$$
\text { int } C_{+}=\left\{u \in C_{+}: u(z)>0 \text { for all } z \in \bar{\Omega}\right\} \text {. }
$$

Let $r \in(1, \infty)$. By $A_{r}: W^{1, r}(\Omega) \rightarrow W^{1, r}(\Omega)^{*}$ we denote the nonlinear operator defined by

$$
\left\langle A_{r}(u), h\right\rangle=\int_{\Omega}|D u|^{r-2}(D u, D h)_{\mathbb{R}^{N}} \mathrm{~d} z \text { for all } u, h \in W^{1, r}(\Omega) .
$$

The next proposition summarizes the main properties of this map (see GasińskiPapageorgiou [12], p. 279).

Proposition 2.1 The operator $A_{r}: W^{1, r}(\Omega) \rightarrow W^{1, r}(\Omega)^{*}$ is bounded (that is, it maps bounded sets to bounded sets), continuous, monotone (hence maximal monotone too) and of type $(S)_{+}$, that is, it has the following property

$$
\begin{aligned}
& \text { "If } u_{n} \stackrel{w}{\rightarrow} u \text { in } W^{1, r}(\Omega) \text { and } \lim _{n \rightarrow \infty}\left\langle A_{r}\left(u_{n}\right), u_{n}-u\right\rangle \leq 0 \text {, then } u_{n} \rightarrow u \text { in } \\
& W^{1, r}(\Omega) \text { ". }
\end{aligned}
$$

For $x \in \mathbb{R}$, we set $x^{ \pm}=\max \{ \pm x, 0\}$. Then, for $u \in W^{1, p}(\Omega)$, we define $u^{ \pm}(z)=$ $u(z)^{ \pm}$for all $z \in \Omega$. We know that

$$
u^{ \pm} \in W^{1, p}(\Omega), \quad|u|=u^{+}+u^{-}, \quad u=u^{+}-u^{-} .
$$

If $h_{1}, h_{2}: \Omega \rightarrow \mathbb{R}$ are two measurable functions such that $h_{1}(z) \leq h_{2}(z)$ for a.a. $z \in \Omega$, then we define the order interval $\left[h_{1}, h_{2}\right]$ in $W^{1, p}(\Omega)$ by

$$
\left[h_{1}, h_{2}\right]=\left\{u \in W^{1, p}(\Omega): h_{1}(z) \leq u(z) \leq h_{2}(z) \text { for a.a. } z \in \Omega\right\} .
$$

Also, we define

$$
\begin{aligned}
& {\left[h_{1}\right)=\left\{u \in W^{1, p}(\Omega): h_{1}(z) \leq u(z) \text { for a.a. } z \in \Omega\right\},} \\
& \left(h_{2}\right]=\left\{v \in W^{1, p}(\Omega): v(z) \leq h_{2}(z) \text { for a.a. } z \in \Omega\right\} .
\end{aligned}
$$


Given a set $S \subseteq W^{1, p}(\Omega)$, we say that $S$ is downward (resp. upward) directed, if for every $u_{1}, u_{2} \in S$ we can find $u \in S$ such that $u \leq u_{1}, u \leq u_{2}$ (resp. $u_{1} \leq u$, $\left.u_{2} \leq u\right)$.

Let $X$ be a Banach space and $\varphi \in C^{1}(X)$. By $K_{\varphi}$ we denote the critical set of $\varphi$, that is,

$$
K_{\varphi}=\left\{u \in X: \varphi^{\prime}(u)=0\right\} .
$$

We say that $\varphi(\cdot)$ satisfies the $C$-condition, if it has the following property

"Every sequence $\left\{u_{n}\right\}_{n \in \mathbb{N}} \subseteq X$ such that

$$
\begin{aligned}
& \left\{\varphi\left(u_{n}\right)\right\}_{n \in \mathbb{N}} \subseteq \mathbb{R} \text { is bounded, } \\
& \left(1+\left\|u_{n}\right\|_{X}\right) \varphi^{\prime}\left(u_{n}\right) \rightarrow 0 \text { in } X^{*} \text { as } n \rightarrow \infty,
\end{aligned}
$$

admits a strongly convergent subsequence”.

For $c \in \mathbb{R}$, let $\varphi^{c}=\{u \in X: \varphi(u) \leq c\}$. Let $Y_{2} \subseteq Y_{1} \subseteq X$. For every $k \in \mathbb{N}_{0}=\mathbb{N} \cup\{0\}$, by $H_{k}\left(Y_{1}, Y_{2}\right)$ we denote the $k^{\text {th }}$ relative singular homology group with integer coefficients for the pair $\left(Y_{1}, Y_{2}\right)$. Suppose that $u \in K_{\varphi}$ is isolated and let $c=\varphi(u)$. Then, the critical groups of $\varphi(\cdot)$ at $u$, are defined by

$$
C_{k}(\varphi, u)=H_{k}\left(\varphi^{c} \cap \mathcal{U}, \varphi^{c} \cap \mathcal{U} \backslash\{u\}\right) \text { for all } k \in \mathbb{N}_{0}
$$

where $\mathcal{U}$ is an open neighborhood of $u$ such that $K_{\varphi} \cap \varphi^{c} \cap \mathcal{U}=\{u\}$.

The excision property of singular homology, implies that this definition is independent of the choice of the isolating neighborhood $\mathcal{U}$.

In the resonant case we will use the spectrum of the operator $u \mapsto-\Delta_{p} u+$ $\xi(z)|u|^{p-2} u$ with the Robin boundary condition. So, for $\xi \in L^{\infty}(\Omega)$ and $\beta \in$ $C^{0, \alpha}(\partial \Omega)(0<\alpha<1), \beta \geq 0$, we consider the following nonlinear eigenvalue problem

$$
\left\{\begin{array}{ll}
-\Delta_{p} u(z)+\xi(z)|u(z)|^{p-2} u(z)=\widehat{\lambda}|u(z)|^{p-2} u(z) & \text { in } \Omega \\
\frac{\partial u}{\partial n_{p}}+\beta(z)|u|^{p-2} u=0 & \text { on } \partial \Omega
\end{array} .\right.
$$

We say that $\widehat{\lambda} \in \mathbb{R}$ is an eigenvalue, if problem (2.1) admits a nontrivial solution $\widehat{u} \in W^{1, p}(\Omega)$, known as an eigenfunction corresponding to the eigenvalue $\widehat{\lambda} \in \mathbb{R}$. The nonlinear regularity theory (see Lieberman [15]) implies that $\widehat{u} \in C^{1}(\bar{\Omega})$. The eigenvalue problem (2.1) was studied by Fragnelli-Mugnai-Papageorgiou [9], who 
established the existence of a smallest eigenvalue $\widehat{\lambda}_{1}(p) \in \mathbb{R}$, which is simple, isolated and admits the following variational characterization

$$
\widehat{\lambda}_{1}(p)=\inf \left[\frac{\gamma_{p}(u)}{\|u\|_{p}^{p}}: u \in W^{1, p}(\Omega), u \neq 0\right]
$$

Here $\gamma_{p}: W^{1, p}(\Omega) \rightarrow \mathbb{R}$ is the $C^{1}$-functional defined by

$$
\gamma_{p}(u)=\|D u\|_{p}^{p}+\int_{\Omega} \xi(z)|u|^{p} \mathrm{~d} z+\int_{\partial \Omega} \beta(z)|u|^{p} \mathrm{~d} \sigma \quad \text { for all } u \in W^{1, p}(\Omega),
$$

with $\sigma(\cdot)$ denoting the $(N-1)$-dimensional Hausdorff (surface) measure on $\partial \Omega$. The infimum in (2.2) is realized on the corresponding one-dimensional eigenspace, the elements of which have fixed sign. By $\widehat{u}_{1}(p)$ we denote the positive, $L^{p}$-normalized (that is, $\left\|\widehat{u}_{1}(p)\right\|_{p}=1$ ) eigenfunction for $\widehat{\lambda}_{1}(p)$. The nonlinear regularity theory and the nonlinear maximum principle, imply that $\widehat{u}_{1}(p) \in \operatorname{int} C_{+}$. Note that if $\xi \geq 0$ and $\xi \not \equiv 0$ or $\beta \not \equiv 0$, then $\widehat{\lambda}_{1}(p)>0$ (see [9]).

We mention that $\widehat{\lambda}_{1}(p)$ is the only eigenvalue with eigenfunctions of fixed sign. All other eigenvalues have nodal (that is, sign-changing) eigenfunctions.

Now we can introduce our hypotheses on the data of problem (1.1).

$\mathrm{H}_{0}: \xi \in L^{\infty}(\Omega), \beta \in C^{0, \alpha}(\partial \Omega), \beta(z) \geq 0$ for all $z \in \partial \Omega$.

Remarks We stress thay $\xi(\cdot)$ is in general sign changing. If $\beta \equiv 0$, then we have a Neumann problem.

For the resonant case, the hypotheses on the reaction $f(z, x)$ are the following (recall that $\left.F(z, x)=\int_{0}^{x} f(z, s) \mathrm{d} s\right)$.

$\mathrm{H}_{1}: f: \Omega \times \mathbb{R} \rightarrow \mathbb{R}$ is a Carathéodory function such that $f(z, 0)=0$ for a.a. $z \in \Omega$ and

(i) $|f(z, x)| \leq a(z)\left[1+|x|^{p-1}\right]$ for a.a. $z \in \Omega$, all $x \in \mathbb{R}$, with $a \in L^{\infty}(\Omega)$;

(ii) $\lim _{x \rightarrow \pm \infty} \frac{p \bar{F}(z, x)}{|x|^{p}}=\widehat{\lambda}_{1}(p)$ uniformly for a.a. $z \in \Omega$;

(iii) there exists $\tau \in(q, p)$ such that

$$
\limsup _{x \rightarrow \pm \infty} \frac{f(z, x) x-p F(z, x)}{|x|^{\tau}} \leq-\gamma_{0}<0 \quad \text { uniformly for a.a. } z \in \Omega
$$

(iv) there exist $\mu \in(1, q)$ and $\delta>0$ such that

$$
c_{0}|x|^{\mu} \leq f(z, x) x \leq \mu F(z, x) \text { for a.a. } z \in \Omega \text {, all }|x| \leq \delta
$$


(v) there exist $\vartheta_{-}<0<\vartheta_{+}$such that for a.a. $z \in \Omega$ we have

$$
f\left(z, \vartheta_{+}\right)-\xi(z) \vartheta_{+}^{p-1} \leq-\widehat{c}_{+}<0<\widehat{c}_{-} \leq f\left(z, \vartheta_{-}\right)+\xi(z)\left|\vartheta_{-}\right|^{p-1}
$$

(vi) for every $\rho>0$, there exists $\widehat{\xi}_{\rho}>0$ such that for a.a. $z \in \Omega$, the function $x \mapsto f(z, x)+\widehat{\xi}_{\rho}|x|^{p-2} x$ is nondecreasing on $[-\rho, \rho]$.

Remarks If $\lim _{x \rightarrow \pm \infty} \frac{f(z, x)}{|x|^{p-2} x}=\widehat{\lambda}_{1}(p)$ uniformly for a.a. $z \in \Omega$, then hypothesis $\mathrm{H}_{1}$ (ii) is satisfied. Therefore we see thay hypothesis $\mathrm{H}_{1}$ (ii) covers the case of problems which are resonant as $x \rightarrow \pm \infty$ with respect to the principal eigenvalue $\widehat{\lambda}_{1}(p)$. In the process of the proof, we will show that

$$
\liminf _{x \rightarrow \pm \infty} \frac{p F(z, x)-\widehat{\lambda}_{1}(p)|x|^{p}}{|x|^{\tau}} \geq \gamma_{1}>0
$$

uniformly for a.a. $z \in \Omega$. This means that the resonance occurs from the right of $\widehat{\lambda}_{1}(p)$ and this makes the energy functional of the problem noncoercive, hence we can not employ the direct method of the calculus of variations. Hypothesis $\mathrm{H}_{1}$ (iv) implies the presence of a concave term near zero. When there is no potential term (that is, $\xi \equiv 0$ ), then hypotheses $\mathrm{H}_{1}(\mathrm{iv})$, (v) dictate an oscillatory behavior for $f(z, \cdot)$ near zero. In the general case the oscillatory behavior concerns the function $x \mapsto$ $f(z, x)-\xi(z)|x|^{p-2} x$. Hypothesis $\mathrm{H}_{1}$ (vi) is essentially a one-sided Hölder condition. It is satisfied if $f(z, \cdot)$ is differentiable for a.a. $z \in \Omega$ and for every $\rho>0$, we can find $\widehat{\xi}_{\rho}>0$ such that $f_{x}^{\prime}(z, x) x^{2} \geq-\widehat{\xi}_{\rho}|x|^{p}$ for a.a. $z \in \Omega$, all $|x| \leq \rho$.

\section{Constant sign solutions}

In this section we produce multiple constant sign solutions.

We start by producing two nontrivial smooth solutions, one positive and the other negative, using only the conditions on $f(z, \cdot)$ near zero.

Proposition 3.1 If hypotheses $\mathrm{H}_{0}, \mathrm{H}_{1}$ (iv), (v), (vi) hold, then problem (1.1) has two constant sign solutions $u_{0} \in \operatorname{int} C_{+}, v_{0} \in-\operatorname{int} C_{+}$and $\vartheta_{-}<v_{0}(z)<0<u_{0}(z)<$ $\vartheta_{+}$for all $z \in \bar{\Omega}$.

Proof First we produce the positive solution.

Let $\eta>\|\xi\|_{\infty}$ (see hypotheses $\mathrm{H}_{0}$ ) and consider the Carathéodory function $k_{+}$: $\Omega \times \mathbb{R} \rightarrow \mathbb{R}$ defined by

$$
k_{+}(z, x)=\left\{\begin{array}{ll}
f\left(z, x^{+}\right)+\eta\left(x^{+}\right)^{p-1} & \text { if } x \leq \vartheta_{+} \\
f\left(z, \vartheta_{+}\right)+\eta \vartheta_{+}^{p-1} & \text { if } \vartheta_{+}<x
\end{array} .\right.
$$


We set $K_{+}(z, x)=\int_{0}^{x} k_{+}(z, s) \mathrm{d} s$ and consider the $C^{1}$-functional $w_{+}$: $W^{1, p}(\Omega) \rightarrow \mathbb{R}$ defined by

$$
w_{+}(u)=\frac{1}{p} \gamma_{p}(u)+\frac{\eta}{p}\|u\|_{p}^{p}+\frac{1}{q}\|D u\|_{q}^{q}-\int_{\Omega} K_{+}(z, u) \mathrm{d} z \text { for all } u \in W^{1, p}(\Omega) .
$$

From (3.1) and since $\eta>\|\xi\|_{\infty}$ and $\beta \geq 0$ (see hypotheses $\mathrm{H}_{0}$ ), we see that $w_{+}(\cdot)$ is coercive. Also, using the Sobolev embedding theorem, we show that $w_{+}(\cdot)$ is sequentially weakly lower semicontinuous. So, by the Weierstrass-Tonelli theorem, we can find $u_{0} \in W^{1, p}(\Omega)$ such that

$$
w_{+}\left(u_{0}\right)=\min \left[w_{+}(u): u \in W^{1, p}(\Omega)\right]
$$

Let $u \in \operatorname{int} C_{+}$and choose $t \in(0,1)$ small such that

$$
0<t u(z) \leq \min \left\{\vartheta_{+}, \delta\right\} \text { for all } z \in \bar{\Omega}
$$

with $\delta>0$ as in hypothesis $\mathrm{H}_{1}$ (iv). We have

$$
\begin{aligned}
w_{+}(t u)= & \frac{t^{p}}{p} \gamma_{p}(u)+\frac{t^{q}}{q}\|D u\|_{q}^{q}-\int_{\Omega} F(x, t u) \mathrm{d} z \quad(\text { see }(3.1),(3.3)) \\
\leq & \frac{t^{q}}{q}\left[\gamma_{p}(u)+\|D u\|_{q}^{q}\right]-\frac{c_{0}}{\mu} t^{\mu}\|u\|_{\mu}^{\mu} \\
& \left(\text { see }(3.3), \text { hypothesis } \mathrm{H}_{1}(\text { iv) and recall that } t \in(0,1), q<p)\right. \\
= & c_{1} t^{q}-c_{2} t^{\mu} \text { for some } c_{1}, c_{2}>0 .
\end{aligned}
$$

Since $\mu<q$ (see $\mathrm{H}_{1}(\mathrm{iv})$ ), choosing $t \in(0,1)$ even smaller if necessary, we have

$$
\begin{aligned}
& w_{+}(t u)<0 \\
& \Rightarrow \quad w_{+}\left(u_{0}\right)<0=w_{+}(0) \quad(\text { see }(3.2)) \\
& \Rightarrow \quad u_{0} \neq 0 .
\end{aligned}
$$

From (3.2) we have

$$
\begin{gathered}
w_{+}^{\prime}\left(u_{0}\right)=0 \\
\Rightarrow\left\langle\gamma_{p}^{\prime}\left(u_{0}\right), h\right\rangle+\int_{\Omega} \eta\left|u_{0}\right|^{p-2} u_{0} h \mathrm{~d} z+\left\langle A_{q}\left(u_{0}\right), h\right\rangle \\
=\int_{\Omega} k_{+}\left(z, u_{0}\right) h \mathrm{~d} z \text { for all } h \in W^{1, p}(\Omega)
\end{gathered}
$$


Evidently

$$
\begin{aligned}
\left\langle\gamma_{p}^{\prime}\left(u_{0}\right), h\right\rangle= & \left\langle A_{p}\left(u_{0}\right), h\right\rangle+\int_{\Omega} \xi(z)\left|u_{0}\right|^{p-2} u_{0} h \mathrm{~d} z+\int_{\partial \Omega} \beta(z)\left|u_{0}\right|^{p-2} u_{0} h \mathrm{~d} z \\
& \text { for all } h \in W^{1, p}(\Omega) .
\end{aligned}
$$

Choosing $h=-u_{0}^{-} \in W^{1, p}(\Omega)$ in (3.4), we obtain

$$
\begin{aligned}
& \gamma_{p}\left(u_{0}^{-}\right)+\eta\left\|u_{0}^{-}\right\|_{p}^{p}+\left\|D u_{0}^{-}\right\|_{q}^{q}=0 \quad(\text { see }(3.1)) \\
& \quad \Rightarrow \quad c_{3}\left\|u_{0}^{-}\right\|^{p} \leq 0 \text { for some } c_{3}>0\left(\text { since } \eta>\|\xi\|_{\infty}\right) \\
& \quad \Rightarrow \quad u_{0} \geq 0, u_{0} \neq 0
\end{aligned}
$$

Next in (3.4) we choose $h=\left[u_{0}-\vartheta_{+}\right]^{+} \in W^{1, p}(\Omega)$. We have

$$
\begin{aligned}
& \left\langle\gamma_{p}^{\prime}\left(u_{0}\right),\left(u_{0}-\vartheta_{+}\right)^{+}\right\rangle+\int_{\Omega} \eta\left|u_{0}\right|^{p-2} u_{0}\left(u_{0}-\vartheta_{+}\right)^{+} \mathrm{d} z+\left\langle A_{q}\left(u_{0}\right),\left(u_{0}-\vartheta_{+}\right)^{+}\right\rangle \\
& =\int_{\Omega}\left[f\left(z, \vartheta_{+}\right)+\eta \vartheta_{+}^{p-1}\right]\left(u_{0}-\vartheta_{+}\right)^{+} \mathrm{d} z \quad(\operatorname{see}(3.1)) \\
& \leq \int_{\Omega}[\xi(z)+\eta] \vartheta_{+}^{p-1}\left(u_{0}-\vartheta_{+}\right)^{+} \mathrm{d} z \quad\left(\text { see hypothesis } \mathrm{H}_{1}(\mathrm{v})\right) \\
& \leq\left\langle\gamma_{p}^{\prime}\left(\vartheta_{+}\right),\left(u_{0}-\vartheta_{+}\right)^{+}\right\rangle \\
& \quad+\int_{\Omega} \eta \vartheta_{+}^{p-1}\left(u_{0}-\vartheta_{+}\right)^{+} \mathrm{d} z+\left\langle A_{q}\left(\vartheta_{+}\right),\left(u_{0}-\vartheta_{+}\right)^{+}\right\rangle \quad(\text { since } \beta \geq 0) \\
& \Rightarrow u_{0} \leq \vartheta_{+} .
\end{aligned}
$$

So, we have proved that

$$
u_{0} \in\left[0, \vartheta_{+}\right], u_{0} \neq 0 .
$$

From (3.5), (3.1) and (3.4) it follows that

$$
u_{0} \in W^{1, p}(\Omega) \text { is a positive solution of }(1.1)
$$

From Proposition 2.10 of Papageorgiou-Rădulescu [18] we have that $u_{0} \in L^{\infty}(\Omega)$. So, we can apply the nonlinear regularity theory of Lieberman [15] and infer that $u_{0} \in C_{+} \backslash\{0\}$. For $\rho=\left\|u_{0}\right\|_{\infty}$, let $\widehat{\xi}_{\rho}>0$ be as postulated by hypothesis $\mathrm{H}_{1}(\mathrm{vi})$. We have

$$
\begin{gathered}
\Delta_{p} u_{0}+\Delta_{q} u_{0} \leq\left[\|\xi\|_{\infty}+\widehat{\xi}_{\rho}\right] u_{0}^{p-1} \text { in } \Omega\left(\text { see hypothesis } \mathrm{H}_{1}(\mathrm{vi})\right) \\
\Rightarrow \quad u_{0} \in \operatorname{int} C_{+} \quad(\text { see Pucci-Serrin }[30], \text { pp. } 111,120)
\end{gathered}
$$


Clearly we can always assume that $\widehat{\xi}_{\rho}>\|\xi\|_{\infty}$. We have

$$
\begin{aligned}
- & \Delta_{p} u_{0}-\Delta_{q} u_{0}+\left[\xi(z)+\widehat{\xi}_{\rho}\right] u_{0}^{p-1} \\
& =f\left(z, u_{0}\right)+\widehat{\xi}_{\rho} u_{0}^{p-1} \\
& \leq f\left(x, \vartheta_{+}\right)+\widehat{\xi}_{\rho} \vartheta_{+}^{p-1}\left(\text { see }(3.5) \text { and hypothesis } \mathrm{H}_{1}(\mathrm{vi})\right) \\
& \leq-\Delta_{p} \vartheta_{+}-\Delta_{q} \vartheta_{+}+\left[\xi(z)+\widehat{\xi}_{\rho}\right] \vartheta_{+}^{p-1} \quad\left(\text { see hypothesis } \mathrm{H}_{1}(\mathrm{v})\right) .
\end{aligned}
$$

On account of hypothesis $\mathrm{H}_{1}(\mathrm{v})$ and using Proposition 2.10 of PapageorgiouRădulescu-Repovš [20], from (3.6) we infer that

$$
u_{0}(z)<\vartheta_{+} \text {for all } z \in \Omega
$$

Suppose that for some $z_{0} \in \partial \Omega$ we have $u_{0}\left(z_{0}\right)=\vartheta_{+}$. Then, from [20] we have $\frac{\partial u_{0}}{\partial n}\left(z_{0}\right)>0$. On the other hand from the Robin boundary condition, we have

$$
\frac{\partial u_{0}}{\partial n}\left(z_{0}\right)=-\beta\left(z_{0}\right) u_{0}\left(z_{0}\right)^{p-1} \leq 0,
$$

a contradiction. Therefore, we conclude that

$$
0<u_{0}(z)<\vartheta_{+} \quad \text { for all } z \in \bar{\Omega} \text {. }
$$

For the negative solution, we introduce the Carathéodory function $k_{-}(z, x)$ defined by

$$
k_{-}(z, x)= \begin{cases}f\left(z, \vartheta_{-}\right)-\eta\left|\vartheta_{-}\right|^{p-1} & \text { if } x<\vartheta_{-} \\ f\left(z,-x^{-}\right)-\eta\left(x^{-}\right)^{p-1} & \text { if } \vartheta_{-} \leq x\end{cases}
$$

We set $K_{-}(z, x)=\int_{0}^{x} k_{-}(z, s) \mathrm{d} s$ and consider the $C^{1}$-functional $w_{-}: W^{1, p}(\Omega) \rightarrow$ $\mathbb{R}$ defined by $w_{-}(u)=\frac{1}{p} \gamma_{p}(u)+\frac{\eta}{p}\|u\|_{p}^{p}+\frac{1}{q}\|D u\|_{q}^{q}-\int_{\Omega} K_{-}(z, u) \mathrm{d} z \quad$ for all $u \in W^{1, p}(\Omega)$.

Using $w_{-}(\cdot),(3.7)$ and the direct method of the calculus of variations, as before, we produce a negative solution $v_{0} \in W^{1, p}(\Omega)$ for problem (1.1) such that

$$
v_{0} \in-\text { int } C_{+}, \quad \vartheta_{-}<v_{0}(z)<0 \quad \text { for all } z \in \bar{\Omega}
$$


Using these two constant sign solutions and activating the asymptotic hypotheses as $x \rightarrow \pm \infty$ (see $\mathrm{H}_{1}$ (ii), (iii)), we can produce two more constant sign smooth solutions localized with respect to $u_{0}$ and $v_{0}$ respectively.

So, let $u_{0} \in$ int $C_{+}$and $v_{0} \in-$ int $C_{+}$be the two solutions from Proposition 3.1. As before, let $\eta>\|\xi\|_{\infty}$ and consider the Carathéodory functions $\widehat{g}_{+}, \widehat{g}_{-}: \Omega \times \mathbb{R} \rightarrow \mathbb{R}$ defined by

$$
\begin{aligned}
& \widehat{g}_{+}(z, x)= \begin{cases}f\left(z, u_{0}(z)\right)+\eta u_{0}(z)^{p-1} & \text { if } x \leq u_{0}(z) \\
f(z, x)+\eta x^{p-1} & \text { if } u_{0}(z)<x\end{cases} \\
& \widehat{g}_{-}(z, x)= \begin{cases}f(z, x)+\eta|x|^{p-2} x & \text { if } x<v_{0}(z) \\
f\left(z, v_{0}(z)\right)+\eta\left|v_{0}(z)\right|^{p-2} v_{0}(z) & \text { if } v_{0}(z) \leq x\end{cases}
\end{aligned}
$$

We set $\widehat{G}_{+}(z, x)=\int_{0}^{x} \widehat{g}_{+}(z, s) \mathrm{d} s$ and $\widehat{G}_{-}(z, x)=\int_{0}^{x} \widehat{g}_{-}(z, s) \mathrm{d} s$ and consider the $C^{1}$-functionals $\widehat{\varphi}_{+}, \widehat{\varphi}_{-}: W^{1, p}(\Omega) \rightarrow \mathbb{R}$ defined by

$$
\begin{aligned}
& \widehat{\varphi}_{+}(u)=\frac{1}{p} \gamma_{p}(u)+\frac{\eta}{p}\|u\|_{p}^{p}+\frac{1}{p}\|D u\|_{q}^{q}-\int_{\Omega} \widehat{G}_{+}(z, u) \mathrm{d} z \\
& \widehat{\varphi}_{-}(u)=\frac{1}{p} \gamma_{p}(u)+\frac{\eta}{p}\|u\|_{p}^{p}+\frac{1}{p}\|D u\|_{q}^{q}-\int_{\Omega} \widehat{G}_{-}(z, u) \mathrm{d} z
\end{aligned}
$$

for all $u \in W^{1, p}(\Omega)$.

Since $u_{0}(z)<\vartheta_{+}$and $\vartheta_{-}<v_{0}(z)$ for all $z \in \bar{\Omega}$ (see Proposition 3.1), we can also introduce the following truncations of $\widehat{g}_{ \pm}(z, \cdot)$

$$
\begin{aligned}
& \widehat{g}_{+}^{*}(z, x)= \begin{cases}\widehat{g}_{+}(z, x) & \text { if } x \leq \vartheta_{+} \\
\widehat{g}_{+}\left(z, \vartheta_{+}\right) & \text {if } \vartheta_{+}<x\end{cases} \\
& \widehat{g}_{-}^{*}(z, x)= \begin{cases}\widehat{g}_{-}\left(z, \vartheta_{-}\right) & \text {if } x<\vartheta_{-} \\
\widehat{g}_{-}(z, x) & \text { if } \vartheta_{-} \leq x\end{cases}
\end{aligned}
$$

Both are Carathéodory functions. We set $\widehat{G}_{+}^{*}(z, x)=\int_{0}^{x} \widehat{g}_{+}^{*}(z, s) \mathrm{d} s$ and $\widehat{G}_{-}^{*}(z, x)=\int_{0}^{x} \widehat{g}_{-}^{*}(z, s) \mathrm{d} s$ and consider the $C^{1}$-functionals $\widehat{\varphi}_{+}^{*}, \widehat{\varphi}_{+}^{*}: W^{1, p}(\Omega) \rightarrow \mathbb{R}$ defined by

$$
\begin{aligned}
& \widehat{\varphi}_{+}^{*}(u)=\frac{1}{p} \gamma_{p}(u)+\frac{\eta}{p}\|u\|_{p}^{p}+\frac{1}{p}\|D u\|_{q}^{q}-\int_{\Omega} \widehat{G}_{+}^{*}(z, u) \mathrm{d} z \\
& \widehat{\varphi}_{-}^{*}(u)=\frac{1}{p} \gamma_{p}(u)+\frac{\eta}{p}\|u\|_{p}^{p}+\frac{1}{p}\|D u\|_{q}^{q}-\int_{\Omega} \widehat{G}_{-}^{*}(z, u) \mathrm{d} z
\end{aligned}
$$

for all $u \in W^{1, p}(\Omega)$. 
From (3.10) and (3.11) and since $\eta>\|\xi\|_{\infty}$, we see that the functionals $\widehat{\varphi}_{+}^{*}, \widehat{\varphi}_{+}^{*}$ are coercive. Moreover, from (3.8), (3.9), (3.10), (3.11), we have that

$$
\left.\widehat{\varphi}_{+}\right|_{\left[0, \vartheta_{+}\right]}=\left.\widehat{\varphi}_{+}^{*}\right|_{\left[0, \vartheta_{+}\right]} \text {and }\left.\widehat{\varphi}_{+}\right|_{\left[0, \vartheta_{+}\right]}=\left.\left(\widehat{\varphi}_{+}^{*}\right)^{\prime}\right|_{\left[0, \vartheta_{+}\right]} \text {. }
$$

Similarly for $\widehat{\varphi}_{-}$and $\widehat{\varphi}_{-}^{*}$ on $\left[\vartheta_{-}, 0\right]$.

Proposition 3.2 If hypotheses $\mathrm{H}_{0}, \mathrm{H}_{1}$ hold, then the functionals $\widehat{\varphi}_{+}, \widehat{\varphi}_{+}$satisfy the C-condition.

Proof We do the proof for $\widehat{\varphi}_{+}(\cdot)$, the proof for $\widehat{\varphi}_{-}(\cdot)$ being similar.

So, we consider a sequence $\left\{u_{n}\right\}_{n \in \mathbb{N}} \subseteq W^{1, p}(\Omega)$ such that

$$
\begin{aligned}
& \left|\widehat{\varphi}_{+}\left(u_{n}\right)\right| \leq c_{4} \text { for some } c_{4}>0, \text { all } n \in \mathbb{N}, \\
& \left(1+\left\|u_{n}\right\|\right) \widehat{\varphi}_{+}^{\prime}\left(u_{n}\right) \rightarrow 0 \text { in } W^{1, p}(\Omega)^{*} \text { as } n \rightarrow \infty
\end{aligned}
$$

From (3.14) we have

$$
\begin{aligned}
& \left.\left|\left\langle\gamma_{p}^{\prime}\left(u_{n}\right), h\right\rangle+\int_{\Omega} \eta\right| u_{n}\right|^{p-2} u_{n} h \mathrm{~d} z+\left\langle A_{q}\left(u_{n}\right), h\right\rangle-\int_{\Omega} \widehat{g}_{+}\left(z, u_{n}\right) h \mathrm{~d} z \mid \leq \frac{\epsilon_{n}\|h\|}{1+\left\|u_{n}\right\|} \\
& \text { for all } h \in W^{1, p}(\Omega), \text { with } \epsilon_{n} \rightarrow 0^{+} .
\end{aligned}
$$

In (3.15) we choose $h=-u_{n}^{-} \in W^{1, p}(\Omega)$. Then, from (3.8) we have

$$
\begin{aligned}
& \gamma_{p}\left(u_{n}^{-}\right)+\eta\left\|u_{n}^{-}\right\|_{p}^{p} \leq c_{5} \text { for some } c_{5}>0, \text { all } n \in \mathbb{N}, \\
& \quad \Rightarrow \quad\left\|u_{n}^{-}\right\|^{p} \leq c_{6} \text { for some } c_{6}>0, \text { all } n \in \mathbb{N}\left(\text { since } \eta>\|\xi\|_{\infty}\right), \\
& \Rightarrow \quad\left\{u_{n}^{-}\right\}_{n \in \mathbb{N}} \subseteq W^{1, p}(\Omega) \text { is bounded. }
\end{aligned}
$$

Next we show that $\left\{u_{n}^{+}\right\} \subseteq W^{1, p}(\Omega)$ is bounded too. We argue by contradiction. So, by passing to a subsequence if necessary, we may assume that

$$
\left\|u_{n}^{+}\right\| \rightarrow+\infty \text { as } n \rightarrow \infty
$$

We set $y_{n}=\frac{u_{n}^{+}}{\left\|u_{n}^{+}\right\|}, n \in \mathbb{N}$. Then $\left\|y_{n}\right\|=1, y_{n} \geq 0$ for all $n \in \mathbb{N}$. We may assume that

$$
y_{n} \stackrel{w}{\rightarrow} y \text { in } W^{1, p}(\Omega) \text { and } y_{n} \rightarrow y \text { in } L^{p}(\Omega) \text { and in } L^{p}(\partial \Omega), y \geq 0 .
$$


From (3.13) and (3.16), we have

$$
\begin{aligned}
& \frac{\widehat{\varphi}_{+}\left(u_{n}^{+}\right)}{\left\|u_{n}^{+}\right\|} \leq \frac{c_{7}}{\left\|u_{n}^{+}\right\|^{p}} \text { for some } c_{7}>0, \text { all } n \in \mathbb{N} \\
& \quad \Rightarrow \quad \frac{1}{p} \gamma_{p}\left(y_{n}\right)+\frac{1}{q} \frac{1}{\left\|u_{n}^{+}\right\|^{p-q}}\left\|D y_{n}\right\|_{q}-\int_{\Omega} \frac{F\left(z, u_{n}^{+}\right)}{\left\|u_{n}^{+}\right\|^{p}} \mathrm{~d} z \\
& \quad \leq \frac{c_{7}}{\left\|u_{n}^{+}\right\|^{p}} \text { for all } n \in \mathbb{N}(\operatorname{see}(3.8)) .
\end{aligned}
$$

Hypotheses $\mathrm{H}_{1}$ (ii), (iii) imply that given $\epsilon>0$, we can find $c_{8}>0$ such that

$$
F(z, x) \leq \frac{1}{p}\left[\widehat{\lambda}_{1}(p)+\epsilon\right] x^{p}+c_{8} \text { for a.a. } z \in \Omega, \text { all } x \geq 0 .
$$

We return to (3.19) and (3.20). We obtain

$$
\frac{1}{p} \gamma_{p}\left(y_{n}\right) \leq \frac{1}{p} \int_{\Omega}\left[\widehat{\lambda}_{1}(p)+\epsilon\right] y_{n}^{p} \mathrm{~d} z+\epsilon_{n}^{\prime} \quad \text { with } \epsilon_{n}^{\prime} \rightarrow 0^{+}
$$

We pass to the limit as $n \rightarrow \infty$ and use (3.18) and the weak lower semicontinuity of $\gamma_{p}(\cdot)$ (since $\gamma_{p}(\cdot)$ is continuous, convex). We obtain

$$
\frac{1}{p} \gamma_{p}(y) \leq \frac{1}{p} \int_{\Omega}\left[\widehat{\lambda}_{1}(p)+\epsilon\right] y^{p} \mathrm{~d} z
$$

Since $\epsilon>0$ is arbitrary, we let $\epsilon \rightarrow 0^{+}$and we have

$$
\begin{aligned}
& \gamma_{p}(y) \leq \widehat{\lambda}_{1}(p)\|y\|_{p}^{p}, \\
& \quad \Rightarrow \quad \gamma_{p}(y)=\widehat{\lambda}_{1}(p)\|y\|_{p}^{p} \quad(\operatorname{see}(2.2)) .
\end{aligned}
$$

From (3.14), (3.16) and (3.8), we have

$$
\begin{aligned}
& \left|\left\langle\gamma_{p}^{\prime}\left(u_{n}^{+}\right), h\right\rangle+\left\langle A_{q}\left(u_{n}^{+}\right), h\right\rangle-\int_{\Omega} f\left(z, u_{n}^{+}\right) h \mathrm{~d} z\right| \leq c_{9}\|h\| \text { for some } c_{9}>0, \text { all } n \in \mathbb{N}, \\
& \quad \Rightarrow\left|\left\langle\gamma_{p}^{\prime}\left(u_{n}^{+}\right), h\right\rangle+\frac{1}{\left\|u_{n}^{+}\right\|^{p-q}}\left\langle A_{q}\left(y_{n}\right), h\right\rangle-\int_{\Omega} \frac{f\left(z, u_{n}^{+}\right)}{\left\|u_{n}^{+}\right\|^{p-1}} h \mathrm{~d} z\right| \\
& \quad \leq \frac{c_{9}\|h\|}{\left\|u_{n}^{+}\right\|^{p-1}} \quad \text { for all } n \in \mathbb{N} .
\end{aligned}
$$

Hypothesis $\mathrm{H}_{1}(\mathrm{i})$ implies that

$$
\left\{\frac{f\left(\cdot, u_{n}^{+}(\cdot)\right)}{\left\|u_{n}^{+}\right\|^{p-1}}\right\}_{n \in \mathbb{N}} \subseteq L^{p^{\prime}}(\Omega) \text { is bounded }\left(p^{\prime}=\frac{p}{p-1}\right)
$$


So, if in (3.22) we choose $h=y_{n}-y \in W^{1, p}(\Omega)$, pass to the limit as $n \rightarrow+\infty$ and use (3.17), (3.18) and (3.23), we obtain

$$
\begin{aligned}
& \lim _{n \rightarrow \infty}\left\langle A_{p}\left(y_{n}\right), y_{n}-y\right\rangle=0, \\
& \quad \Rightarrow \quad y_{n} \rightarrow y \text { in } W^{1, p}(\Omega) \text { and so }\|y\|=1, y \geq 0 .
\end{aligned}
$$

From (3.21) and (3.24), we infer that

$$
y=c \widehat{u}_{1}(p) \in \operatorname{int} C_{+} \quad \text { with } c>0 .
$$

Then it follows that

$$
\begin{aligned}
& u_{n}^{+}(z) \rightarrow+\infty \text { for a.a. } z \in \Omega, \\
\Rightarrow & \limsup _{n \rightarrow \infty} \frac{f\left(z, u_{n}^{+}(z)\right) u_{n}^{+}(z)-p F\left(z, u_{n}^{+}(z)\right)}{u_{n}^{+}(z)^{\tau}} \leq-\gamma_{0}<0 \\
& \text { for a.a. } z \in \Omega\left(\text { see hypothesis } \mathrm{H}_{1}(\mathrm{iii})\right), \\
\Rightarrow & \liminf _{n \rightarrow \infty} \int_{\Omega} \frac{p F\left(z, u_{n}^{+}\right)-f\left(z, u_{n}^{+}\right) u_{n}^{+}}{\left\|u_{n}^{+}\right\|^{\tau}} \geq \gamma_{1}>0 \\
& \text { (using Fatou's lemma and (3.24)). }
\end{aligned}
$$

From (3.13), (3.16), (3.8) and (3.17), we have

$$
\begin{aligned}
-\gamma_{p}\left(y_{n}\right)-\frac{p}{q} \frac{1}{\left\|u_{n}^{+}\right\|^{p-q}}\left\|D y_{n}\right\|_{q}^{q}+\int_{\Omega} \frac{p F\left(z, u_{n}^{+}\right)}{\left\|u_{n}^{+}\right\|^{p}} \mathrm{~d} z \leq \frac{c_{10}}{\left\|u_{n}^{+}\right\|^{p}} \\
\text { for some } c_{10}>0, \text { all } n \in \mathbb{N} .
\end{aligned}
$$

On the other hand, from (3.15) with $h=u_{n}^{+} \in W^{1, p}(\Omega),(3.16)$ and (3.8), we have

$$
\begin{aligned}
& \gamma_{p}\left(y_{n}\right)+\frac{1}{\left\|u_{n}^{+}\right\| p-q}\left\|D y_{n}\right\|_{q}^{q}-\int_{\Omega} \frac{f\left(z, u_{n}^{+}\right) u_{n}^{+}}{\left\|u_{n}^{+}\right\|^{p}} \mathrm{~d} z \leq \frac{c_{11}}{\left\|u_{n}^{+}\right\|^{p}} \\
& \quad \text { for some } c_{11}>0, \text { all } n \in \mathbb{N} .
\end{aligned}
$$

We add (3.26) and (3.27). Then we have

$$
\frac{1}{\left\|u_{n}^{+}\right\|^{p-\tau}} \int_{\Omega} \frac{p F\left(z, u_{n}^{+}\right)-f\left(z, u_{n}^{+}\right) u_{n}^{+}}{\left\|u_{n}^{+}\right\|^{\tau}} \mathrm{d} z \leq\left(\frac{p}{q}-1\right) \frac{1}{\left\|u_{n}^{+}\right\|^{p-q}}\left\|D y_{n}\right\|_{q}^{q}+\frac{c_{12}}{\left\|u_{n}^{+}\right\|^{p}}
$$

for some $c_{12}>0$, all $n \in \mathbb{N}$,

$$
\Rightarrow \quad \int_{\Omega} \frac{p F\left(z, u_{n}^{+}\right)-f\left(z, u_{n}^{+}\right) u_{n}^{+}}{\left\|u_{n}^{+}\right\|^{\tau}} \mathrm{d} z \leq\left(\frac{p}{q}-1\right) \frac{1}{\left\|u_{n}^{+}\right\|^{\tau-q}}\left\|D y_{n}\right\|_{q}^{q}+\epsilon_{n}^{*}
$$


for all $n \in \mathbb{N}$, with $\epsilon_{n}^{*} \rightarrow 0^{+}$,

$$
\Rightarrow \limsup _{n \rightarrow \infty} \int_{\Omega} \frac{p F\left(z, u_{n}^{+}\right)-f\left(z, u_{n}^{+}\right) u_{n}^{+}}{\left\|u_{n}^{+}\right\|^{\tau}} \mathrm{d} z \leq 0
$$

(recall that $q<\tau$ and see (3.17)).

Comparing (3.28) and (3.25), we have a contradiction. Hence

$$
\begin{aligned}
& \left\{u_{n}^{+}\right\}_{n \in \mathbb{N}} \subseteq W^{1, p}(\Omega) \text { is bounded } \\
& \quad \Rightarrow \quad\left\{u_{n}\right\}_{n \in \mathbb{N}} \subseteq W^{1, p}(\Omega) \text { is bounded (see (3.16)). }
\end{aligned}
$$

We may assume that

$$
u_{n} \stackrel{w}{\rightarrow} u \text { in } W^{1, p}(\Omega) \text { and } u_{n} \rightarrow u \text { in } L^{p}(\Omega) \text { and in } L^{p}(\partial \Omega) .
$$

In (3.15) we choose $h=u_{n}-u \in W^{1, p}(\Omega)$, pass to the limit as $n \rightarrow \infty$ and use (3.29). Then

$$
\begin{aligned}
& \lim _{n \rightarrow \infty}\left[\left\langle A_{p}\left(u_{n}\right), u_{n}-u\right\rangle+\left\langle A_{q}\left(u_{n}\right), u_{n}-u\right\rangle\right]=0, \\
& \quad \Rightarrow \quad \limsup _{n \rightarrow \infty}\left[\left\langle A_{p}\left(u_{n}\right), u_{n}-u\right\rangle+\left\langle A_{q}(u), u_{n}-u\right\rangle\right] \leq 0 \quad\left(\text { since } A_{q}(\cdot)\right. \text { is monotone) } \\
& \Rightarrow \quad \limsup _{n \rightarrow \infty}\left\langle A_{p}\left(u_{n}\right), u_{n}-u\right\rangle \leq 0 \quad(\operatorname{see}(3.29)) \\
& \Rightarrow \quad u_{n} \rightarrow u \text { in } W^{1, p}(\Omega) \text { (see Proposition 2.1) }
\end{aligned}
$$

This proves that $\widehat{\varphi}_{+}(\cdot)$ satisfies the C-condition.

In a similar fashion, we show that $\widehat{\varphi}_{-}(\cdot)$ satisfies the $\mathrm{C}$-condition.

Proposition 3.3 If hypotheses $\mathrm{H}_{0}, \mathrm{H}_{1}$ hold, the $\widehat{\varphi}_{ \pm}\left(t \widehat{u}_{1}(p)\right) \rightarrow-\infty$ as $t \rightarrow \pm \infty$.

Proof For a.a. $z \in \Omega$ and all $x>0$, we have

$$
\begin{aligned}
\frac{\mathrm{d}}{\mathrm{d} x}\left(\frac{F(z, x)}{x^{p}}\right) & =\frac{f(z, x) x^{p}-p F(z, x) x^{p-1}}{x^{2 p}} \\
& =\frac{f(z, x) x-p F(z, x)}{x^{p+1}} .
\end{aligned}
$$

On account of hypothesis $\mathrm{H}_{1}(\mathrm{iii})$, we can find $\gamma_{2} \in\left(0, \gamma_{0}\right)$ and $M>0$ such that

$$
f(z, x) x-p F(z, x) \leq-\gamma_{2} x^{\tau} \text { for a.a. } z \in \Omega \text {, all } x \geq M \text {. }
$$


We return to (3.30) and use (3.31). Then

$$
\begin{aligned}
& \frac{\mathrm{d}}{\mathrm{d} x}\left(\frac{F(z, x)}{x^{p}}\right) \leq-\frac{\gamma_{2} x^{\tau}}{x^{p+1}}=-\frac{\gamma_{2}}{x^{p-\tau+1}} \text { for a.a. } z \in \Omega, \text { all } x \geq M, \\
& \Rightarrow \frac{F(z, x)}{x^{p}}-\frac{F(z, y)}{y^{p}} \leq \frac{\gamma_{2}}{p-\tau}\left(\frac{1}{x^{p-\tau}}-\frac{1}{y^{p-\tau}}\right) \text { for a.a. } z \in \Omega, \text { all } x \geq y \geq M .
\end{aligned}
$$

We pass to the limit as $x \rightarrow+\infty$ and use hypothesis $\mathrm{H}_{1}(\mathrm{ii})$. Then

$$
\begin{aligned}
& \frac{\widehat{\lambda}_{1}(p)}{p}-\frac{F(z, y)}{y^{p}} \leq-\frac{\gamma_{2}}{p-\tau} \frac{1}{y^{p-\tau}} \text { for a.a. } z \in \Omega, \text { all } y \geq M, \\
& \Rightarrow \quad \frac{p F(z, y)-\widehat{\lambda}_{1}(p) y^{p}}{y^{\tau}} \geq \frac{\gamma_{2}}{p-\tau} \text { for a.a. } z \in \Omega, \text { all } y \geq M .
\end{aligned}
$$

We infer that

$$
\liminf _{x \rightarrow+\infty} \frac{p F(z, x)-\widehat{\lambda}_{1}(p) x^{p}}{x^{\tau}} \geq \gamma_{3}>0 \text { uniformly for a.a. } z \in \Omega .
$$

Then we have

$$
\begin{aligned}
& \widehat{\varphi}_{+}\left(t \widehat{u}_{1}(p)\right)=\frac{\widehat{\lambda}_{1}(p)}{p}\left\|t \widehat{u}_{1}(p)\right\|_{p}^{p}+\frac{t^{q}}{q}\left\|D \widehat{u}_{1}\right\|_{q}^{q}-\int_{\Omega} F\left(z, t \widehat{u}_{1}(p)\right) \mathrm{d} z \\
& \Rightarrow p \widehat{\varphi}_{+}\left(t \widehat{u}_{1}(p)\right)=\int_{\Omega} \frac{\widehat{\lambda}_{1}(p)\left(t \widehat{u}_{1}(p)\right)-p F\left(z, t \widehat{u}_{1}(p)\right)}{t^{\tau}} t^{\tau} \mathrm{d} z+\frac{t^{q}}{q}\left\|D \widehat{u}_{1}\right\|_{q}^{q} .
\end{aligned}
$$

Using (3.32) and the fact that $\tau>q$ (see hypothesis $\mathrm{H}_{1}(\mathrm{iii})$ ), we infer that

$$
\widehat{\varphi}_{+}\left(t \widehat{u}_{1}(p)\right) \rightarrow-\infty \text { as } t \rightarrow+\infty
$$

In a similar fashion we show that

$$
\widehat{\varphi}_{-}\left(t \widehat{u}_{1}(p)\right) \rightarrow-\infty \text { as } t \rightarrow-\infty \text {. }
$$

Using (3.8) and (3.9), we can easily check that

$$
K_{\widehat{\varphi}_{+}} \subseteq\left[u_{0}\right) \cap \operatorname{int} C_{+} \text {and } K_{\widehat{\varphi}_{-}} \subseteq\left(v_{0}\right] \cap\left(-\operatorname{int} C_{+}\right)
$$

We may assume that

$$
K_{\widehat{\varphi}_{+}} \cap\left[0, \vartheta_{+}\right]=\left\{u_{0}\right\} \text { and } K_{\widehat{\varphi}_{-}} \cap\left[\vartheta_{-}, 0\right]=\left\{v_{0}\right\}
$$

Otherwise we already have two more constant sign solutions, one positive different from $u_{0}$ in $\left[u_{0}, \vartheta_{+}\right] \cap$ int $C_{+}$and the other negative different from $v_{0}$ in $\left[\vartheta_{-}, v_{0}\right] \cap$ $\left(-\right.$ int $\left.C_{+}\right)$. So, we are done. 
Clearly the functionals $\widehat{\varphi}_{+}^{*}$ and $\widehat{\varphi}_{-}^{*}$ are coercive (see (3.10) and (3.11)) and sequentially weakly lower semicontinuous. Moreover, we can easily check that

$$
K_{\widehat{\varphi}_{+}^{*}} \subseteq\left[u_{0}, \vartheta_{+}\right] \cap \operatorname{int} C_{+} \text {and } K_{\widehat{\varphi}_{-}^{*}} \subseteq\left[\vartheta_{-}, v_{0}\right] \cap\left(-\operatorname{int} C_{+}\right)
$$

The Weierstrass-Tonelli theorem, together with (3.34), (3.35) and (3.12) and the fact that $u_{0} \in$ int $_{C^{1}(\bar{\Omega})}\left[0, \vartheta_{+}\right]$(that is, the interior in $C^{1}(\bar{\Omega})$ of $\left[0, \vartheta_{+}\right] \cap C^{1}(\bar{\Omega})$ ), $v_{0} \in$ int $_{C^{1}(\bar{\Omega})}\left[-\vartheta_{-}, 0\right]$ (that is, the interior in $C^{1}(\bar{\Omega})$ of $\left[\vartheta_{-}, 0\right] \cap C^{1}(\bar{\Omega})$ ), imply that

$$
u_{0} \text { is a local } C^{1}(\bar{\Omega})-\text { minimizer of } \widehat{\varphi}_{+},
$$

and

$$
v_{0} \text { is a local } C^{1}(\bar{\Omega})-\text { minimizer of } \widehat{\varphi}_{-} \text {. }
$$

From Papageorgiou-Rădulescu [18] (see also Bai-Gasiński-Winkert-Zeng [4] for a nonsmooth version), we have

$$
u_{0} \text { is a local } W^{1, p}(\Omega)-\text { minimizer of } \widehat{\varphi}_{+},
$$

and

$$
v_{0} \text { is a local } W^{1, p}(\Omega)-\text { minimizer of } \widehat{\varphi}_{-} .
$$

Note that we may say that the sets

$$
K_{\widehat{\varphi}_{+}} \text {and } K_{\widehat{\varphi}_{-}} \text {are finite }
$$

otherwise on account of (3.35), (3.8), (3.9) we already have a sequence of distinct positive solutions bigger than $u_{0}$ and a sequence of distinct negative solutions smaller than $v_{0}$ and so we are done. From (3.36), (3.37), (3.38) and Theorem 5.7.6, p. 449, of Papageorgiou-Rădulescu-Repovš, we see that we can find $\rho \in(0,1)$ small such that

$$
\begin{aligned}
& \widehat{\varphi}_{+}\left(u_{0}\right)<\inf \left[\widehat{\varphi}_{+}(u):\left\|u-u_{0}\right\|=\rho\right]=\widehat{m}_{+}, \\
& \widehat{\varphi}_{-}\left(v_{0}\right)<\inf \left[\widehat{\varphi}_{-}(v):\left\|v-v_{0}\right\|=\rho\right]=\widehat{m}_{-} .
\end{aligned}
$$

Now we are ready to produce two more constant sign solutions for problem (1.1).

Proposition 3.4 If hypotheses $\mathrm{H}_{0}, \mathrm{H}_{1}$ hold, then problem (1.1) has two more constant sign solutions $\widehat{u} \in \operatorname{int} C_{+}, u_{0} \leq \widehat{u}, u_{0} \neq \widehat{u}, \widehat{v} \in-\operatorname{int} C_{+}, \widehat{v} \leq v_{0}, \widehat{v} \neq v_{0}$. 
Proof Propositions 3.2 and 3.3 and (3.39), (3.40) permit the use of the mountain pass theorem on the functionals $\widehat{\varphi}_{+}, \widehat{\varphi}_{-}$. Hence we can find $\widehat{u} \in K_{\hat{\varphi}_{+}} \subseteq\left[u_{0}\right) \cap \operatorname{int} C_{+}$, $\widehat{v} \in K_{\widehat{\varphi}_{-}} \subseteq\left(v_{0}\right] \cap\left(-\right.$ int $\left.C_{+}\right)$(see (3.33)) such that

$$
\widehat{m}_{+} \leq \widehat{\varphi}_{+}(\widehat{u}) \text { and } \widehat{m}_{-} \leq \widehat{\varphi}_{-}(\widehat{v})
$$

From (3.39), (3.40), (3.41) we infer that

$$
\begin{gathered}
u_{0} \leq \widehat{u}, u_{0} \neq \widehat{u}, \\
\widehat{v} \leq v_{0}, \widehat{v} \neq v_{0} .
\end{gathered}
$$

\section{Extremal constant sign solutions}

In this section we show that problem (1.1) has extremal constant sign solutions, that is, there exist a smallest positive solution and a biggest negative solution. Using these extremal solutions, in the next section, we will be able to produce a nodal (signchanging) solution.

Let $S_{+}$(resp. $S_{-}$) be the set of positive (resp. negative) solutions of (1.1). In Sect. 3, we saw that

$$
\varnothing \neq S_{+} \subseteq \text { int } C_{+} \text {and } \varnothing \neq S_{-} \subseteq-\operatorname{int} C_{+}
$$

Proposition 4.1 If hypotheses $\mathrm{H}_{0}, \mathrm{H}_{1}$ hold, then problem (1.1) admits a smallest positive solution $u_{*} \in$ int $C_{+}$and a biggest negative solution.

Proof From Papageorgiou-Rădulescu-Repovš [19] (see the proof of Proposition 7), we know that $S_{+}$is downward directed. So, using Lemma 3.10, p. 178, of HuPapageorgiou [14], we can find a decreasing sequence $\left\{u_{n}\right\}_{n \in \mathbb{N}} \subseteq S_{+}$such that

$$
\inf _{n \geq 1} u_{n}=\inf S_{+}
$$

We have

$$
\begin{aligned}
& \left\langle\gamma_{p}^{\prime}\left(u_{n}\right), h\right\rangle+\left\langle A_{q}\left(u_{n}\right), h\right\rangle=\int_{\Omega} f\left(z, u_{n}\right) h \mathrm{~d} z \text { for all } h \in W^{1, p}(\Omega), \text { all } n \in \mathbb{N} \\
& 0 \leq u_{n} \leq u_{1} \text { for all } n \in \mathbb{N} .
\end{aligned}
$$


From (4.1) (with $h=u_{n} \in W^{1, p}(\Omega)$ ), (4.2) and hypothesis $\mathrm{H}_{1}(\mathrm{i})$ it follows that $\left\{u_{n}\right\}_{n \geq 1} \subseteq W^{1, p}(\Omega)$ is bounded. So, we may assume that

$$
u_{n} \stackrel{w}{\rightarrow} u_{*} \text { in } W^{1, p}(\Omega) \text { and } u_{n} \rightarrow u_{*} \text { in } L^{p}(\Omega) \text { and in } L^{p}(\partial \Omega)
$$

In (4.1) we choose $h=u_{n}-u_{*} \in W^{1, p}(\Omega)$, pass to the limit as $n \rightarrow \infty$ and use (4.3). Then, as in the proof of Proposition 3.2, we obtain

$$
\begin{aligned}
& \underset{n \rightarrow \infty}{\limsup }\left\langle A_{p}\left(u_{n}\right), u_{n}-u_{*}\right\rangle \leq 0 \\
& \quad \Rightarrow \quad u_{n} \rightarrow u_{*} \text { in } W^{1, p}(\Omega) \text { (see Proposition 2.1). }
\end{aligned}
$$

So, passing to the limit as $n \rightarrow \infty$ in (4.1) and using (4.4), we have that

$$
\left\langle\gamma_{p}^{\prime}\left(u_{*}\right), h\right\rangle+\left\langle A_{q}\left(u_{*}\right), h\right\rangle=\int_{\Omega} f\left(z, u_{*}\right) h \mathrm{~d} z \text { for all } h \in W^{1, p}(\Omega) .
$$

If we show that $u_{*} \neq 0$, then $u_{*} \in S_{+}$and so $u_{*}=\inf S_{+}$.

On account of hypotheses $\mathrm{H}_{1}$ (i), (iv), we have

$$
f(z, x) \geq c_{0}|x|^{\mu}-c_{13}|x|^{r} \quad \text { for a.a. } z \in \Omega \text {, all } x \in \mathbb{R} \text {, with } c_{13}>0, r \in\left(p, p^{*}\right) .
$$

The unilateral growth restriction on $f(z, \cdot)$ leads to the following auxiliary Robin problem

$$
\left\{\begin{array}{ll}
-\Delta_{p} u-\Delta_{q} u+|\xi(z)||u|^{p-2} u=c_{0}|u|^{\mu-2} u-c_{13}|u|^{r-2} u & \text { in } \Omega \\
\frac{\partial u}{\partial n_{p q}}+\beta(z)|u|^{p-2} u=0 & \text { on } \partial \Omega
\end{array} .\right.
$$

From Proposition 12 of Papageorgiou-Vetro-Vetro [27], we know that this problem has a unique positive solution $\widetilde{u} \in$ int $C_{+}$and since the equation is odd, $\widetilde{v}=-\widetilde{u} \in$ -int $C_{+}$is the unique negative solution of (4.6).

Claim. $\tilde{u} \leq u$ for all $u \in S_{+}$and $v \leq \tilde{v}$ for all $v \in S_{-}$.

Let $u \in S_{+}$and consider the Carathéodory function $k_{+}: \Omega \times \mathbb{R} \rightarrow \mathbb{R}$ defined by

$$
k_{+}(z, x)= \begin{cases}c_{0}\left(x^{+}\right)^{\mu-1}-c_{13}\left(x^{+}\right)^{r-1} & \text { if } x \leq u(z) \\ c_{0} u(z)^{\mu-1}-c_{13} u(z)^{r-1} & \text { if } u(z)<x\end{cases}
$$


We set $K_{+}(z, x)=\int_{0}^{x} k_{+}(z, s) \mathrm{d} s$ and consider the $C^{1}$-functional $\psi_{+}$: $W^{1, p}(\Omega) \rightarrow \mathbb{R}$ defined by

$$
\psi_{+}(u)=\frac{1}{p} \widehat{\gamma}_{p}(u)+\frac{1}{q}\|D u\|_{q}^{q}-\int_{\Omega} K_{+}(z, u) \mathrm{d} z \text { for all } u \in W^{1, p}(\Omega)
$$

with $\widehat{\gamma}_{p} \in C^{1}\left(W^{1, p}(\Omega)\right)$ being defined by

$$
\widehat{\gamma}_{p}(u)=\|D u\|_{p}^{p}+\int_{\Omega}|\xi(z) \| u|^{p} \mathrm{~d} z+\int_{\partial \Omega} \beta(z)|u|^{p} \mathrm{~d} \sigma \quad \text { for all } u \in W^{1, p}(\Omega) .
$$

Note that $\psi_{+}(\cdot)$ is coercive (see (4.7)). Indeed, for every $v \in W^{1, p}(\Omega)$ we have that

$$
\begin{aligned}
\int_{\Omega} K_{+}(z, v) \mathrm{d} z & =\int_{\{v \leq u\}}\left[\frac{c_{0}}{\mu}\left(v^{+}\right)^{\mu}-\frac{c_{13}}{r}\left(v^{+}\right)^{r}\right] \mathrm{d} z+\int_{\{u<v\}}\left[c_{0} u^{\mu-1}-c_{13} u^{r-1}\right](v-u) \mathrm{d} z \\
& \leq \widehat{c}_{1}[1+\|v\|] \text { for some } \widehat{c}_{1}>0, \\
& \Rightarrow-\int_{\Omega} K_{+}(z, v) \mathrm{d} z \geq-\widehat{c}_{1}[1+\|v\|] .
\end{aligned}
$$

From this we infer the coercivity of $\psi_{+}(\cdot)$ since $p>1$.

Also $\psi_{+}(\cdot)$ is sequentially weakly lower semicontinuous. So, we can find $\tilde{u}_{0} \in$ $W^{1, p}(\Omega)$ such that

$$
\psi_{+}\left(\tilde{u}_{0}\right)=\inf \left[\psi_{+}(u): u \in W^{1, p}(\Omega)\right] .
$$

Since $\mu<q<p$, it follows that

$$
\begin{gathered}
\psi_{+}\left(\tilde{u}_{0}\right)<0=\psi_{+}(0), \\
\Rightarrow \quad \tilde{u}_{0} \neq 0 .
\end{gathered}
$$

Using (4.7) and the nonlinear regularity theory (see [15]), we obtain

$$
K_{\psi_{+}} \subseteq[0, u] \cap C_{+}
$$

From (4.8), (4.9) it follows that

$$
\begin{aligned}
& \tilde{u}_{0} \in[0, u], \tilde{u}_{0} \neq 0 \\
& \quad \Rightarrow \quad \tilde{u}_{0}=\tilde{u} \in \operatorname{int} C_{+}(\operatorname{see}(4.7)) \\
& \Rightarrow \tilde{u} \leq u \text { for all } u \in S_{+}(\operatorname{see}(4.9))
\end{aligned}
$$

Similarly we show that $v \leq \tilde{v}$ for all $v \in S_{-}$. This proves the Claim. 
From (4.4) and the Claim, we have $\tilde{u} \leq u_{*}$. Hence $u_{*} \neq 0$ and so $u_{*} \in S_{+}$, $u_{*}=\inf S_{+}$.

Similarly, we prove the existence of $v_{*} \in S_{-}$and $v \leq v_{*}$ for all $v \in S_{-}$. We point out that now $S_{-}$is upward directed.

\section{Nodal solutions}

In this section, using the extremal constant sign solutions $u_{*} \in \operatorname{int} C_{+}$and $v_{*} \in$ -int $C_{+}$produced in Proposition 4.1, we show the existence of a nodal (sign-changing) solution. The idea is simple. We focus on the order interval $\left[v_{*}, u_{*}\right]$ and using a combination of tools and techniques we show that the problem has a solution in $\left[v_{*}, u_{*}\right]$ distinct from $0, u_{*}, v_{*}$. Then, on account of the extremality of $u_{*}, v_{*}$, such a solution will be nodal.

To this end, let $\eta>\|\xi\|_{\infty}$ and consider the Carathéodory function $l: \Omega \times \mathbb{R} \rightarrow \mathbb{R}$ defined by

$$
l(z, x)= \begin{cases}f\left(z, v_{*}(z)\right)+\eta\left|v_{*}(z)\right|^{p-2} v_{*}(z) & \text { if } x<v_{*}(z) \\ f(z, x)+\eta|x|^{p-2} x & \text { if } v_{*}(z) \leq x \leq u_{*}(z) \\ f\left(z, u_{*}(z)\right)+\eta u_{*}(z)^{p-1} & \text { if } u_{*}(z)<x\end{cases}
$$

Also, we consider the positive and negative truncations of $l(z, \cdot)$, namely the Carathéodory functions $l_{ \pm}: \Omega \times \mathbb{R} \rightarrow \mathbb{R}$ defined by

$$
l_{ \pm}(z, x)=l\left(z, \pm x^{ \pm}\right)
$$

We set $L(z, x)=\int_{0}^{x} l(z, s) \mathrm{d} s, L_{ \pm}(z, s)=\int_{0}^{x} l_{ \pm}(z, s) \mathrm{d} s$ and consider the $C^{1}$ functionals $\lambda, \lambda_{ \pm}: W^{1, p}(\Omega) \rightarrow \mathbb{R}$ defined by

$$
\begin{gathered}
\lambda(u)=\frac{1}{p}\left[\gamma_{p}(u)+\eta\|u\|_{p}^{p}\right]+\frac{1}{q}\|D u\|_{q}^{q}-\int_{\Omega} L(z, u) \mathrm{d} z, \\
\lambda_{ \pm}(u)=\frac{1}{p}\left[\gamma_{p}(u)+\eta\|u\|_{p}^{p}\right]+\frac{1}{q}\|D u\|_{q}^{q}-\int_{\Omega} L_{ \pm}(z, u) \mathrm{d} z,
\end{gathered}
$$

for all $u \in W^{1, p}(\Omega)$.

Using (5.1), (5.2) and the nonlinear regularity theory (see [15]), we show that

$$
\begin{aligned}
& K_{\lambda} \subseteq\left[v_{*}, u_{*}\right] \cap C^{1}(\bar{\Omega}), \\
& K_{\lambda_{+}} \subseteq\left[0, u_{*}\right] \cap C_{+}, \quad K_{\lambda_{-}} \subseteq\left[v_{*}, 0\right] \cap\left(-C_{+}\right)
\end{aligned}
$$


From (5.4) and the extremality of $u_{*}$ and $v_{*}$, we conclude that

$$
K_{\lambda_{+}}=\left\{0, u_{*}\right\}, \quad K_{\lambda_{-}}=\left\{0, v_{*}\right\} .
$$

Proposition 5.1 If hypotheses $\mathrm{H}_{0}, \mathrm{H}_{1}$ hold, then problem (1.1) admits a nodal solution $y_{0} \in C^{1}(\bar{\Omega})$ such that $v_{*} \leq y_{0} \leq u_{*}$.

Proof Clearly $\lambda_{+}$is coercive (see (5.1), (5.2)). Also it is sequentially weakly lower semicontinuous. So, we can find $\tilde{u}_{*} \in W^{1, p}(\Omega)$ such that

$$
\lambda_{+}\left(\tilde{u}_{*}\right)=\min \left[\lambda_{+}(u): u \in W^{1, p}(\Omega)\right] .
$$

On account of hypothesis $\mathrm{H}_{1}$ (iv), we have

$$
\begin{aligned}
\lambda_{+}\left(\tilde{u}_{*}\right)<0=\lambda_{+}(0), \\
\Rightarrow \quad \tilde{u}_{*} \neq 0, \\
\Rightarrow \quad \tilde{u}_{*}=u_{*}(\operatorname{see}(5.6) \text { and }(5.5)) .
\end{aligned}
$$

Since $\left.\lambda\right|_{C_{+}}=\left.\lambda_{+}\right|_{C_{+}}$and $u_{*} \in$ int $C_{+}$, it follows that

$$
\begin{aligned}
& u_{*} \text { is a local } C^{1}(\bar{\Omega})-\text { minimizer of } \lambda(\cdot), \\
& \quad \Rightarrow \quad u_{*} \text { is a local } W^{1, p}(\Omega)-\text { minimizer of } \lambda(\cdot) \text { (see [18], [4]). }
\end{aligned}
$$

Similarly, using this time $\lambda_{-}(\cdot)$ we obtain that

$$
v_{*} \text { is a local } W^{1, p}(\Omega)-\text { minimizer of } \lambda(\cdot) .
$$

We may assume that $\lambda\left(v_{*}\right) \leq \lambda\left(u_{*}\right)$, the analysis is similar if the opposite inequality holds, using (5.8) instead of (5.7). From (5.1) and (5.3) we see that we may assume that $K_{\lambda}$ is finite (otherwise, we already have a whole sequence of distinct smooth nodal solutions in $\left.\left[v_{*}, u_{*}\right]\right)$.

Then from (5.7) and using Theorem 5.7.6, p. 449, of Papageorgiou-RădulescuRepovš [21], we can find $\rho \in(0,1)$ small such that

$$
\lambda\left(v_{*}\right) \leq \lambda\left(u_{*}\right)<\inf \left[\lambda(u):\left\|u-u_{*}\right\|=\rho\right]=m_{\rho} .
$$

Since $\lambda(\cdot)$ is coercive (see (5.1)), it satisfies the C-condition. This fact and (5.9), permit the use of the mountain pass theorem. So, we can find $y_{0} \in W^{1, p}(\Omega)$ such that

$$
y_{0} \in K_{\lambda} \subseteq\left[v_{*}, u_{*}\right] \cap C^{1}(\bar{\Omega}) \quad(\operatorname{see}(5.3)), \quad m_{\rho} \leq \lambda\left(y_{0}\right) \quad(\text { see }(5.9)) .
$$


Evidently $y_{0} \notin\left\{v_{*}, u_{*}\right\}$ (see (5.9), (5.10)). Also from Theorem 6.5.8, p. 527, of Papageorgiou-Rădulescu-Repovš [21], we have

$$
C_{1}\left(\lambda, y_{0}\right) \neq 0
$$

On the other hand, from hypothesis $\mathrm{H}_{1}$ (iv) and Proposition 3.7 of PapageorgiouRădulescu [17], we have that

$$
C_{k}(\lambda, 0)=0 \text { for all } k \in \mathbb{N}_{0}
$$

Comparing (5.11) and (5.12), we see that $y_{0} \neq 0$ and so $y_{0} \in C^{1}(\bar{\Omega})$ is a nodal solution of (1.1) and $v_{*} \leq y_{0} \leq u_{*}$.

So, summarizing the situation for the resonant case, we can state the following multiplicity theorem for problem (1.1).

Theorem 5.2 If hypotheses $\mathrm{H}_{0}, \mathrm{H}_{1}$ hold, then problem (1.1) has at least five nontrivial solutions $u_{0}, \widehat{u} \in \operatorname{int} C_{+}, u_{0} \leq \widehat{u}, u_{0} \neq \widehat{u}, v_{0}, \widehat{v} \in-\operatorname{int} C_{+}, \widehat{v} \leq v_{0}, v_{0} \neq \widehat{v}$, $y_{0} \in C^{1}(\bar{\Omega})$ nodal, $v_{0} \leq y_{0} \leq u_{0}$.

\section{Superlinear problem}

In this section we examine what happens when the reaction $f(z, \cdot)$ is $(p-1)$ superlinear as $x \rightarrow \pm \infty$. The precise hypotheses on the reaction $f(z, x)$ are the following:

$\mathrm{H}_{1}^{\prime}: f: \Omega \times \mathbb{R} \rightarrow \mathbb{R}$ is a Carathéodory function such that $f(z, 0)=0$ for a.a. $z \in \Omega$ and

(i) $|f(z, x)| \leq a(z)\left[1+|x|^{r-1}\right]$ for a.a. $z \in \Omega$, all $x \in \mathbb{R}$, with $a \in L^{\infty}(\Omega)$, $p<r<p^{*}$

(ii) $\lim _{x \rightarrow \pm \infty} \frac{F(z, x)}{|x|^{p}}=+\infty$ uniformly for a.a. $z \in \Omega$;

(iii) there exists $\tau \in\left(\max \left\{1, \frac{N}{p}\right\}(r-p), p^{*}\right), \tau>q$, such that

$$
0<\widehat{\gamma_{0}} \leq \liminf _{x \rightarrow \pm \infty} \frac{f(z, x) x-p F(z, x)}{|x|^{\tau}} \text { uniformly for a.a. } z \in \Omega
$$

(iv) there exist $\mu \in(1, q)$ and $\delta>0$ such that

$$
c_{0}|x|^{\mu} \leq f(z, x) x \leq \mu F(z, x) \text { for a.a. } z \in \Omega, \text { all }|x| \leq \delta
$$


(v) there exist $\vartheta_{-}<0<\vartheta_{+}$such that for a.a. $z \in \Omega$ we have

$$
f\left(z, \vartheta_{+}\right)-\xi(z) \vartheta_{+}^{p-1} \leq-\widehat{c}_{+}<0<\widehat{c}_{-} \leq f\left(z, \vartheta_{-}\right)+\xi(z)\left|\vartheta_{-}\right|^{p-1}
$$

(vi) for every $\rho>0$, there exists $\widehat{\xi}_{\rho}>0$ such that for a.a. $z \in \Omega$, the function $x \mapsto f(z, x)+\widehat{\xi}_{\rho}|x|^{p-2} x$ is nondecreasing on $[-\rho, \rho]$.

Remarks So now compared with hypotheses $\mathrm{H}_{1}$, the conditions determining the behavior of $f(z, \cdot)$ near zero remain the same (see hypotheses $\mathrm{H}_{1}^{\prime}(\mathrm{iv}),(\mathrm{v}),(\mathrm{vi})$ ) and change the asymptotic conditions as $x \rightarrow \pm \infty$ (see hypotheses $\mathrm{H}_{1}^{\prime}$ (ii), (iii)). Hypotheses $\mathrm{H}_{1}^{\prime}$ (ii), (iii) imply that for a.a. $z \in \Omega, f(z, \cdot)$ is $(p-1)$-superlinear, but need not satisfy the usual in superlinear problems Ambrosetti-Rabinowitz condition (the ARcondition for short). Our framework incorporates also superlinear nonlinearities with "slower" growth as $x \rightarrow \pm \infty$. For example, if $\xi \equiv 0$ (no potential term), then the function

$$
f(x)= \begin{cases}|x|^{\mu-2} x-2|x|^{\vartheta-2} x & \text { if }|x| \leq 1 \\ |x|^{p-2} x[\ln |x|-1] & \text { if } 1<|x|\end{cases}
$$

wth $\mu<q<p, \vartheta>\mu$, satisfies hypotheses $\mathrm{H}_{1}^{\prime}$ but fails to satisfy the AR-condition.

We argue as in the resonant case. In this case, Proposition 3.2 (the C-condition for the functionals $\widehat{\varphi}_{+}, \widehat{\varphi}_{-}$), is proved as in the Claim in the proof of Proposition 2 in Papageorgiou-Scapellato [24]. Moreover, in this case Proposition 3.3, is an immediate consequence of hypothesis $\mathrm{H}_{1}^{\prime}$ (ii). Since the conditions near zero are the same, the rest of the results (Proposition 3.4 and those in Sect. 4), remain valid and so finally we can state the following multiplicity theorem for the superlinear problem.

Theorem 6.1 If hypotheses $\mathrm{H}_{0}, \mathrm{H}_{1}^{\prime}$ hold, then problem (1.1) has at least five nontrivial solutions $u_{0}, \widehat{u} \in \operatorname{int} C_{+}, u_{0} \leq \widehat{u}, u_{0} \neq \widehat{u}, v_{0}, \widehat{v} \in-\operatorname{int} C_{+}, \widehat{v} \leq v_{0}, v_{0} \neq \widehat{v}$, $y_{0} \in C^{1}(\bar{\Omega})$ nodal, $v_{0} \leq y_{0} \leq u_{0}$.

Remark We stress that in both Theorems 5.2 and 6.1 the solutions are ordered.

\section{The $(p, 2)$-equation}

In this section we show that when $q=2$, we can slightly improve the two multiplicity theorems (Theorem 5.2 and Theorem 6.1). Our work in this section is also related to the recent paper of Vetro [33] on semilinear equations driven by the Robin Laplacian plus an indefinite potential. The author proves a multiplicity result using the reduction technique of Amann [1]. 
So, now the problem under consideration is the following

$$
\begin{cases}-\Delta_{p} u(z)-\Delta u(z)+\xi(z)|u(z)|^{p-2} u(z)=f(z, u(z)) & \text { in } \Omega \\ \frac{\partial u}{\partial n_{p 2}}+\beta(z)|u|^{p-2} u=0 & \text { on } \partial \Omega\end{cases}
$$

with $2<p$.

Let $a: \mathbb{R}^{N} \rightarrow \mathbb{R}^{N}$ be defined by

$$
a(y)=|y|^{p-2} y+y .
$$

Evidently

$$
\operatorname{div} a(D u)=\Delta_{p} u+\Delta u \text { for all } u \in W^{1, p}(\Omega)
$$

Note that $a \in C^{1}\left(\mathbb{R}^{N}, \mathbb{R}^{N}\right)$ and we have

$$
\nabla a(y)=|y|^{p-2}\left[\mathrm{id}+(p-2) \frac{y \otimes y}{|y|^{2}}\right]+\mathrm{id} \text { for all } y \in \mathbb{R}^{N}
$$

We have

$$
(\nabla a(y) \vartheta, \vartheta)_{\mathbb{R}^{N}} \geq|\vartheta|^{2} \text { for all } y, \vartheta \in \mathbb{R}^{N}
$$

So, invoking the tangency principle of Pucci-Serrin [30] (Theorem 2.5.2, p. 35) and using Proposition 2.10 of [20], we obtain

$$
u_{0}(z)<\widehat{u}(z) \text { for all } z \in \bar{\Omega}
$$

In a similar fashion we also have

$$
\begin{aligned}
& \widehat{v}(z)<v_{0}(z) \text { for all } z \in \bar{\Omega} \\
& \quad v_{0}(z)<y_{0}(z)<u_{0}(z) \text { for all } z \in \bar{\Omega} .
\end{aligned}
$$

Therefore for problem (7.1) we can have the following slightly improved version of Theorems 5.2 and 6.1 .

Theorem 7.1 If hypotheses $\mathrm{H}_{0}, \mathrm{H}_{1}$ (with $q=2$ ) or hypotheses $\mathrm{H}_{0}, \mathrm{H}_{1}^{\prime}$ (with $q=2$ ) hold, then problem (7.1) has at least five nontrivial solutions $u_{0}, \widehat{u} \in \operatorname{int} C_{+}, \widehat{u}-u_{0} \in$ int $C_{+}, v_{0}, \widehat{v} \in-\operatorname{int} C_{+}, v_{0}-\widehat{v} \in \operatorname{int} C_{+}, y_{0} \in C^{1}(\bar{\Omega})$ nodal, $y_{0} \in \operatorname{int}_{C^{1}(\bar{\Omega})}\left[v_{0}, u_{0}\right]$. 
Acknowledgements The authors wish to thank the two anonymous reviewers for their remarks and criticisms which helped them to improve the presentation.

Funding Open access funding provided by Università degli Studi di Catania within the CRUI-CARE Agreement.

\section{Compliance with ethical standards}

Conflict of interest All authors have declare that they have no conflict of interest.

Open Access This article is licensed under a Creative Commons Attribution 4.0 International License, which permits use, sharing, adaptation, distribution and reproduction in any medium or format, as long as you give appropriate credit to the original author(s) and the source, provide a link to the Creative Commons licence, and indicate if changes were made. The images or other third party material in this article are included in the article's Creative Commons licence, unless indicated otherwise in a credit line to the material. If material is not included in the article's Creative Commons licence and your intended use is not permitted by statutory regulation or exceeds the permitted use, you will need to obtain permission directly from the copyright holder. To view a copy of this licence, visit http://creativecommons.org/licenses/by/4.0/.

\section{References}

1. Amann, H.: Saddle points and multiple solutions of differential equations. Math. Z. 169, 127-166 (1979)

2. Amster, P.: Multiple solutions for an elliptic system with indefinite Robin boundary conditions. Adv. Nonlinear Anal. 8(1), 603-614 (2019)

3. Bahrouni, A., Rădulescu, V.D., Repovš, D.D.: Double phase transonic flow problems with variable growth: nonlinear patterns and stationary waves. Nonlinearity 32, 2481-2495 (2019)

4. Bai, Y., Gasiński, L., Winkert, P., Zeng, S.: $W^{1, p}$ versus $C^{1}$ : the nonsmooth case involving critical growth. Bull. Math. Sci. 10, 2050009 (2020)

5. Benci, V., D'Avenia, P., Fortunato, D., Pisani, L.: Solitons in several dimensions: Derrick's problem and infinitely many solutions. Arch. Rat. Mech. Anal. 154, 297-324 (2000)

6. Candito, P., Gasiński, L., Livrea, R.: Three solutions for parametric problems with nonhomogeneous $(a, 2)$-type differential operators and reaction terms sublinear at zero. J. Math. Anal. Appl. 480, 123398 (2019)

7. Cherfils, L., Il'yasov, Y.: On the stationary solutions of generalized reaction diffusion equations with p\&q- Laplacian. Commun. Pure Appl. Anal. 4, 9-22 (2005)

8. Filippakis, M., Papageorgiou, N.S.: Resonant $(p, q)$-equations with Robin boundary condition. Electr. J. Differ. Equ. 2018, 01 (2018)

9. Fragnelli, G., Mugnai, D., Papageorgiou, N.S.: The Brezis-Oswald result for quasilinear Robin problems. Adv. Nonlinear Stud. 16, 603-622 (2016)

10. Gasiński, L., Papageorgiou, N.S.: Dirichlet $(p, q)$-equations at resonance. Dis. Cont. Dyn. Syst. A 34, 2037-2060 (2014)

11. Gasiński, L., Papageorgiou, N.S.: Multiple solutions for asymptotically $(p-1)$-homogeneous $p$ Laplacian equations. J. Funct. Anal. 262, 2403-2435 (2012)

12. Gasiński, L., Papageorgiou, N.S.: Exercises in Analysis. Part 2: Nonlinear Analysis. Springer, Cham (2016) 
13. Gasiński, L., Winkert, P.: Constant sign solutions for double phase problems with superlinear nonlinearity. Nonlin. Anal. 195, 111739 (2020)

14. Hu, S., Papageorgiou, N.S.: Handbook of Multivalued Analysis, vol. I. Kluwer Academic Publishers, Dordrecht (1997)

15. Lieberman, G.: The natural generalization of the natural conditions of Ladyzhenskaya and Ural'tseva for elliptic equations. Commun. Partial Differ. Equ. 16, 311-361 (1991)

16. Li, F., Rong, T., Liang, Z.: Multiple positive solutions for a class of (2, p)-Laplacian equation. J. Math. Phys. 59, 121506 (2018)

17. Papageorgiou, N.S., Rădulescu, V.D.: Coercive and noncoercive nonlinear Neumann problems with indefinite potential. Forum Math. 28, 545-571 (2016)

18. Papageorgiou, N.S., Rădulescu, V.D.: Nonlinear nonhomogeneous Robin problems with superlinear reaction term. Adv. Nonlinear Stud. 16, 737-764 (2016)

19. Papageorgiou, N.S., Rădulescu, V.D., Repovš, D.: Positive solutions for perturbations of the Robin eigenvalue problem plus and indefinite potential. Disc. Cont. Dyn. Syst. A 37, 2589-2618 (2017)

20. Papageorgiou, N.S., Rădulescu, V.D., Repovš, D.: Positive solutions for nonlinear nonhomogeneous parametric Robin problems. Forum Math. 30, 553-580 (2018)

21. Papageorgiou, N.S., Rădulescu, V.D., Repovš, D.: Nonlinear Analysis-Theory and Methods. Springer, Switzerland (2019)

22. Papageorgiou, N.S., Rădulescu, V.D., Repov̌̌, D.: Double-phase problems and a discontinuity property of the spectrum. Proc. Am. Math. Soc. 147(7), 2899-2910 (2019)

23. Papageorgiou, N.S., Rădulescu, V.D., Repovš, D.: Existence and multiplicity of solutions for doublephase Robin problems. Bull. Lond. Math. Soc. 52(3), 546-560 (2020)

24. Papageorgiou, N.S., Scapellato, A.: Concave-convex problems for the Robin $p$-Laplacian plus an indefinite potential. Mathematics 8, 421 (2020)

25. Papageorgiou, N.S., Scapellato, A.: Constant sign and nodal solutions for parametric $(p, 2)$-equations. Adv. Nonlin. Anal. 9, 448-476 (2020)

26. Papageorgiou, N.S., Vetro, C., Vetro, F.: Multiple solutions with sign information for a class of coercive ( $p, 2)$-equations. Bull. Malays. Math. Sci. Soc. 43, 2343-2371 (2020)

27. Papageorgiou, N.S., Vetro, C., Vetro, F.: Parametric dependence for the positive solutions of nonlinear, nonhomogeneous Robin problems. Rev. Real Acad. Cienc. Exactas Fis. Nat. Ser. A Mat. 114, 45 (2020)

28. Papageorgiou, N.S., Zhang, C.: Noncoercive resonant $(p, 2)$-equations with concave terms. Adv. Nonlinear Anal. 9(1), 228-249 (2020)

29. Pei, R., Zhang, J.: Nontrivial solution for asymmetric $(p, 2)$-Laplacian Dirichlet problem. Bound. Value Probl. 2014, 241 (2014)

30. Pucci, P., Serrin, J.: The Maximum Principle. Birkhäuser, Basel (2007)

31. Sun, M.: Multiplicity of solutions for a class of quasilinear elliptic equations at resonance. J. Math. Anal. Appl. 386, 661-668 (2012)

32. Vetro, C.: Perturbed eigenvalue problems for the $p$-Laplacian plus an indefinite potential. Anal. Math. Phys. 10(69), 1-34 (2020)

33. Vetro, C.: Semilinear Robin problems driven by the Laplacian plus an indefinite potential. Complex Var. Elliptic Equ. 65, 573-587 (2020)

34. Zhikov, V.V.: On variational problems and nonlinear elliptic equations with nonstandard growth conditions. J. Math. Sci. 173, 463-570 (2011)

Publisher's Note Springer Nature remains neutral with regard to jurisdictional claims in published maps and institutional affiliations. 